\section{Hartnäckiger Rückenschmerz bei Älteren}

Deutlich mehr als die Hälfte der Patienten, die im Rahmen einer Studie ihren Hausarzt mit Rückenbeschwerden aufsuchten, klagten nach drei Monaten noch immer über Schmerzen. Die niederländischen Kollegen identifizierten eine Reihe von Risikofaktoren.

$\mathrm{n}$ dieser prospektiven Kohortenstudie wurden 675 Patienten (durchschnittliches Alter: 66,4 Jahre), die in den vorausgehenden sechs Monaten keinen Arzt wegen Rückenschmerzen konsultiert hatten, beobachtet. $\mathrm{Zu}$ Studienbeginn wurden die Teilnehmer untersucht und dokumentierten ihre Beschwerden in einem Fragebogen. Nach sechs Wochen und drei Monaten machten sie erneut Angaben über ihr Befinden. Bei ihrem ersten Arztbesuch hatten die Patienten auf der NRS (Numeric Rating Scale 0-10) einen durchschnittlichen Schmerzgrad von 5,2 angegeben. Nach sechs Wochen lag dieser bei den 639 antwortenden Teilnehmern bei 3,7 und nach drei Monaten $(n=626)$ bei 3,6. Bei der Bewertung der Leistungseinbußen durch die Rückenbeschwerden mit dem RDQ (Roland Disability Questionnaire 0-24) verringerte sich der Ausgangswert von 9,8 innerhalb von drei Monaten auf 7,8. Nach sechs Wochen hatten $64 \%$ der Patienten allerdings immer noch Schmerzen, nach drei Monaten lag die Quote bei
$61 \%$. Zu diesem Zeitpunkt versuchten noch $39 \%$ der Patienten, ihren Rücken mit Analgetika zu beruhigen. Nur 26\% der Probanden suchten in dieser Zeit erneut ihren Arzt auf.

In der multiplen Regressionsanalyse fand sich ein signifikanter Zusammenhang der langwierigen Rückenbeschwerden mit folgenden Kriterien: bestehender Rückenschmerz von 6-12 Wochen und mehr, besonders starke Beschwerden, häufige Rückenschmerzepisoden in der Vorgeschichte, fehlendes Ausstrahlen des Schmerzes in den Bereich unterhalb des Knies, mehrere Komorbiditäten, negative Erwartungen des Patienten sowie eine verlängerte Dauer beim Timed Up \& Go-Test.

Fazit: Wie in Studien mit jüngeren Probanden gingen auch bei den Älteren dieser Untersuchung die Schmerzen zunächst relativ zügig zurück, nach sechs Wochen allerdings nur noch sehr langsam. Insgesamt war der Umfang der Schmerzminderung mit $15 \%$ in den ers-

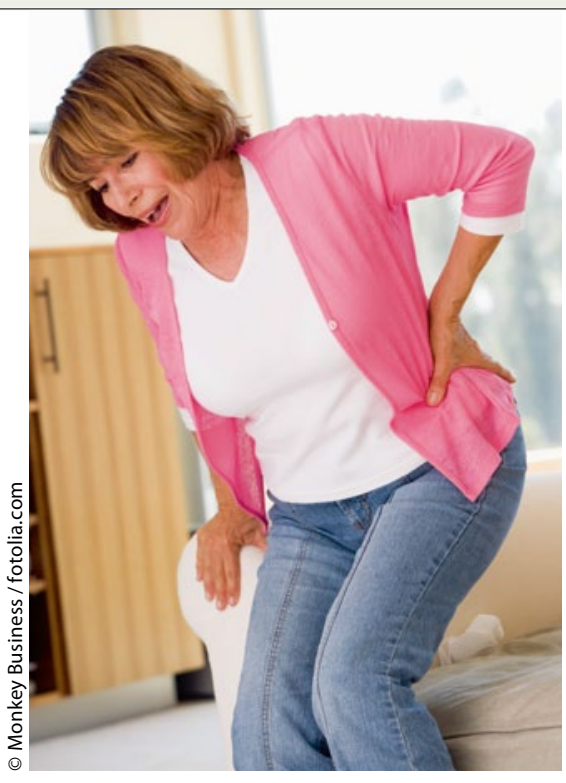

Verschiedene Altersleiden können langwierige Rückenbeschwerden begünstigen.

ten sechs Wochen im Vergleich zu anderen Untersuchungen jedoch gering. Die Autoren gehen davon aus, dass der Hausarzt durch die Kenntnis möglicher Risikofaktoren die Prognose seiner Rückenschmerzpatienten besser abschätzen kann. Bevor die genannten Faktoren allerdings Eingang in die praktische Bewertung finden können, müssen sie in weiteren Untersuchungen bestätigt werden.

Christine Starostzik

Scheele J et al. Course and prognosis of older back pain patients in general practice: a prospective cohort study. Pain. 2013;154(6):951-7.

\section{Retardiertes Gabapentin bei neuropathischen Schmerzen}

\begin{abstract}
Untersucht wurde die neue galenische Aufbereitung von Gabapentin, die nur einmal täglich eingenommen werden soll. Die Resorption erfolgt verzögert im Magen. Die Ergebnisse: Das Präparat ist gut verträglich und sicher.
\end{abstract}

Es wurden zwei placebokontrollierte Studien mit insgesamt 723 Patienten durchgeführt. Aufnahmekriterium war eine postherpetische Neuralgie mit einem Schmerzniveau von über 4 aus 10 auf der numerischen Analogskala. Hauptzielkriterien waren Nebenwirkungen und die Sicherheit der Medikation. Der Gesamtanteil von Patienten mit Nebenwirkungen (vor allem Müdigkeit und Benommenheit) lag bei Gabapentin bei
$54 \%$ und bei Placebo bei $42 \%$. Ernsthafte Nebenwirkungen hatten $2 \%$ der Gabapentin- und 3\% der Placebogruppe. Aber die Medikation reduzierte die Schmerzen deutlich. Daraus folgern die Autoren, dass diese Aufbereitung von Gabapentin sicher und gut verträglich ist.

Irving GA, Sweeney M. Tolerability and safety of gastroretentive once-daily gabapentin tablets for the treatment of postherpetic neuralgia. J Pain Res. 2012;5:203-8.
Kommentar von Stefan Evers: Gabapentin ist nach wie vor eines der wichtigsten Medikamente in der Therapie neuropathischer Schmerzen. Nur musste man bei der bisherigen Aufbereitung 3-mal täglich dosieren. Dies mindert die Compliance der Patienten. Insofern ist es begrüßenswert, dass jetzt eine "retardierte" Form entwickelt wurde, die sicher und effektiv in der Behandlung von Schmerzen nach einem Herpes zoster ist. Vermutlich gelten diese Ergebnisse auch für andere Schmerzformen.

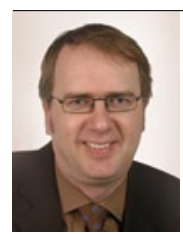

Prof. Dr. med. Stefan Evers Universitätsklinikum Münster 